\title{
Les enjeux et les défis de l'Internet des Objets (IdO)
}

\section{Issues and Challenges of the Internet of Things (IOT)}

\author{
Imad Saleh ${ }^{1}$ \\ ${ }^{1}$ Laboratoire Paragraphe Université Paris 8, imad.saleh@univ-paris8.fr
}

\begin{abstract}
RÉSUMÉ. Dans cet article, nous présentons : 1) les concepts de l'Internet des objets (IdO), 2) Les données, source de création de la valeur, 3) Les enjeux socio-économiques de l'IdO, 4) Les Enjeux pour les entreprises et les chercheurs, 5) Et la dernière partie a pour objectif de décrire les thématiques de la revue de l'internet des objets (IdO).

ABSTRACT. In this paper, we present: 1) the concepts of the Internet of things (IoT), 2) the data as a source of value creation, 3) the socio-economic challenges of loT, 3) The challenges for companies and researchers, 5) and the last part has as objective to describe the themes of the review of the internet of objects (IoT).

MOTS-CLÉS. internet des objets, internet of Everything, Big Data, normes, science de l'information, trace, données privées, données publiques, éthique.

KEYWORDS. internet of things, internet of everything, Big Data, normes, track, private data, Information science, ethics.
\end{abstract}

Aujourd'hui, Internet se transforme progressivement en un HyperRéseau, comme un réseau formé par des multitudes de connexions entre des Artefacts (physiques, documentaires), des acteurs (biologiques, algorithmiques), des écritures et des concepts (linked data, metadata, ontologies, folksonomie), appelé «Internet of Things (IoT) Internet des objets (IdO)», connectant des milliards d'êtres humains, mais aussi des milliards d'objets. Il devient l'outil le plus puissant jamais inventé par l'homme pour créer, modifier, et partager les informations. Cette transformation montre l'évolution du réseau d'internet : d'un réseau des calculateurs vers à un réseau d'ordinateurs personnels, et puis vers un réseau nomade intégrant les technologies des communications [CHA 2012]. Les développements des technologies Machine-toMachine (M2M) pour le contrôle de machine à distance et aussi l'apparition dans l'année 2000 d'IP (Internet Protocole) sur les réseaux mobiles cellulaires ont accéléré l'évolution de M2M vers l'IdO [WOO 2011].

Un objet connecté doit être adopté à un usage, il a une certaine forme d'intelligence, une capacité de recevoir, de transmettre des données avec des logiciels grâce aux capteurs embarqués [ROX 2017].

Un objet à trois éléments clés :

- Les données produites ou reçues, stockées ou transmises.

- Les algorithmes pour traiter ces données.

-L'écosystème dans lequel il va réagir et s'intégrer.

Les propriétés d'usage d'un objet :

- Ergonomie (utilisabilité, maniabilité, ...).

- Esthétisme (formes/couleurs/sons/sensations, ...).

- Usage (histoire culturelle, profil, matrice sociale, ...).

- Méta-Morphisme (adaptabilité, personnalisation, modulation, ...).

\section{Définition}

Le CERP-IdO « Cluster des projets européens de recherche sur l'Internet des objets » définit l'Internet des objets comme : " une infrastructure dynamique d'un réseau global. Ce réseau global a des capacités d'auto- configuration basée sur des standards et des protocoles de communication interopérables. Dans ce réseau, les objets physiques et virtuels ont des identités, des attributs physiques, des personnalités 
virtuelles et des interfaces intelligentes, et ils sont intégrés au réseau d'une façon transparente » [CLU 2010].

Cette définition montre les deux aspects de l'IdO : temporel et spatial qui permettent aux personnes de se connecter de n'importe où à n'importe quel moment à travers des objets connectés [CHA 2012] (figure 1) (Smartphone, tablettes, capteurs, caméras de vidéosurveillance, etc.). Un objet connecté a une valeur lorsqu'il est connecté à d'autres objets et briques logicielles, par exemple : une montre connectée n'a d'intérêt qu'au sein d'un écosystème orienté santé/bien-être, qui va bien au-delà de connaitre l'heure.

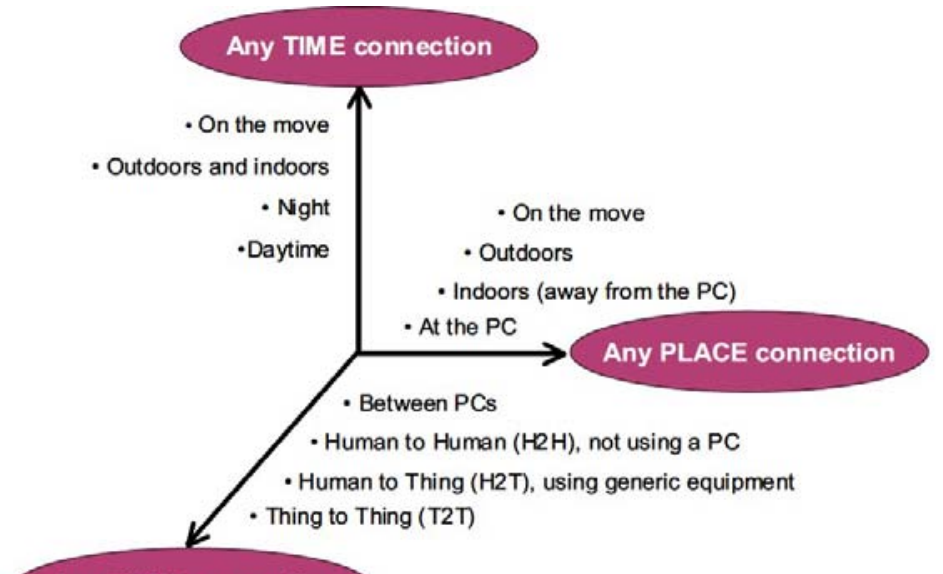

Any THING connection

Figure 1. Une nouvelle dimension pour I'IdO (Source ITU 2005 [INT 2005] tirée de [CHA 2012])

«L'émergence de cet "Internet des Objets », qui tend à brouiller les frontières entre l'ordinateur et les produits du quotidien, s'explique par deux facteurs majeurs: la banalisation des ressources informatiques et l'adoption des services Web par les utilisateurs » (figure 2) [THE 2013].

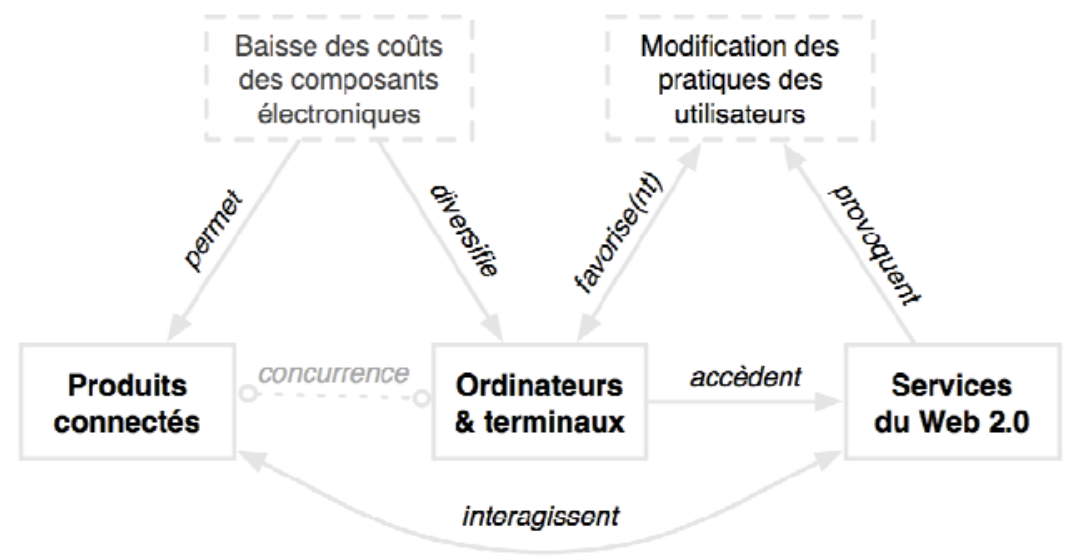

Figure 2. Facteurs déclencheurs de l'Internet des Objets [THE 2013]

L'Internet des objets doit être pensé pour un usage facile et une manipulation sécurisée pour éviter des menaces et risques potentiels, tout en masquant la complexité technologique sous-jacente.

Certains chercheurs parlent des « hyper objets » [MAV 2003] capables de mutualiser leurs ressources afin d'effectuer des tâches du quotidien, ils sont reliés par des "liens invisibles » au sein d'un même écosystème.

Dans ce contexte, certains chercheurs comme [WEI 1993] ont déjà envisagé de l'informatique ubiquitaire là où "les technologies les plus profondes sont celles qui sont devenues invisibles. Celles qui, nouées ensemble, forment le tissu de notre vie quotidienne au point d'en devenir indissociables » [WEI 1991, p. 94]. 
La communication entre les objets passe par des identifications connues entre eux. Un objet doit avoir un ou plusieurs identifiants (codes-barres) pour être reconnu par un autre et établir la connexion. Le système GS1 (mettre la référence) a proposé une technologie basée sur des étiquettes RFID (Radio Frequency Identification) associant de manière unique, les informations logistiques liées à un objet, à une adresse URL. Google a proposé le projet Physical Web pour associer de manière unique une adresse URL à un objet ${ }^{1}$. L'omniprésence dans notre vie des objets hétérogènes, mobiles et fragiles pose le problème des modèles de confiance adaptés à cet écosystème complexe et fragile ?. Derrière ces technologies apparaît la bataille pour les normes et standards pour l'IdO entre les entreprises géantes d'internet car chacune souhaite imposer ses technologies.

\section{Les données, source de création de la valeur}

Les données jouent un rôle important pour le développement économique des entreprises. Les analyses des «traces numériques » laissées par l'usage d'internet, permettent de personnaliser le service adapté au profil de l'utilisateur, mais aussi, adapté à l'endroit où se trouve l'usager. Les données produites par les objets connectés peuvent fournir des informations sur les habitudes, les compétences ou les relations des usagers.

Les entreprises numériques ont déjà compris l'importance de contrôler les traces des usagers et d'en accroître leur nombre. Certaines entreprises proposent de contrôler ces données et d'identifier les objets. C'est un défi majeur pour l'IdO qui en même temps soulève des problèmes d'éthique et des problèmes d'authenticité pour l'usager des données produites. Dans ce contexte, plus les objets sont dotés d'algorithmes intelligents pour percevoir et agir, plus ils deviennent autonomes, ils augmentent les problèmes liés à la vie privée. Il devient donc important de développer des technologies pour permettre aux objets une auto-immunité contre les codes malveillants ou des pénétrations non autorisées pour éviter la propagation des données ou des codes erronés.

Les données peuvent être localisées et stockées dans une base de données centralisée ou globalisées, dans des bases de données distribuées grâce aux technologies du cloud computing.

Ainsi, le degré de confiance en l'IdO et son acceptation sont des conditions primordiales pour la mise en place de mesures adéquates de protection des données personnelles et de la vie privée. En effet, il est important de prévoir lors de la conception des éléments de l'IdO, l'ensemble des exigences des usagers et la sécurité des données [COM 2009]. Il existe des solutions basées sur la cryptographie ou sur la gestion de clés à base de prédistribution qui pourraient accommoder les contraintes de ressources des objets [CHA 2012]. Néanmoins, des questions sont soulevées sur la robustesse et la solidité de telles solutions à un IdO comportant potentiellement des millions d'objets.

Aujourd'hui, on sait où, quand, qui produit les données structurées ou non (Big Data). Ces précisions font le succès des tweets qui, en plus du contenu textuel du message, encapsulent des informations spatiotemporelles et par croisement avec le profil de l'auteur, ajoutent des informations sur un réseau social particulier. Cela soulève des problèmes épistémologiques et méthodologiques importants [BOY 2014, RIE 2012] concernant la multiplication des « micro-interprétations » des données et à leur représentation. La Data Science peut fournir une base nécessaire pour le Big Data. Elle est basée sur des techniques telles que le data mining, machine learning, visual analytics, le cloud, le parallel computing et la récupération de l'information [ADI 2017, MAS 2015]. Elle s'intéresse au traitement des données et au processus de visualisation dans des domaines à flux permanent des données tels que la santé, le transport, des assurances, etc.

Certaines données peuvent être utiles ou non, mais le grand défi se situe au niveau de la contextualisation de ces données pour leur donner un sens pour créer de la valeur pour les usagers et les entreprises. Les données transmises par l'IdO sont des matériaux bruts et non traités, celles-ci sont

\footnotetext{
${ }^{1}$ https://google.github.io/physical-web/

(C) 2017 ISTE OpenScience - Published by ISTE Ltd. London, UK - openscience.fr
} 
utiles lorsqu'elles sont combinées et traitées pour former des informations qui ont un sens. Il est nécessaire de filtrer les données pour connaître leur utilité en sachant que des données peuvent être utiles pour certains processus, mais elles ne le sont pas pour d'autres. Les rôles des algorithmes intelligents peuvent venir au secours pour filtrer les pertinences des données. Néanmoins, il est donc difficile de transformer systématiquement cette immensité de données en informations et par la suite en connaissances utilisables dans la vie quotidienne. Une approche consiste à enrichir sémantiquement ces données à travers des ontologies pour faciliter leur réutilisation et permettre la mise en œuvre des mécanismes de raisonnement [SEY et al. 2015]. De plus si les connaissances sont complétées par les expériences alors les données sont transformées en savoirs et enrichissent les compétences de l'écosystème de l'IdO. Les données générées ou produites par l'IdO soulèvent la question concernant leurs propriétaires et le droit sur elles.

\section{IdO vers l'internet of Everything (IoE)}

Après la société Cisco $^{2}$ [CIS 2013], la convergence entre les réseaux des personnes, des processus, des données et des objets, l'IdO va vers l'internet of Everything (IoE), ou « Internet du Tout connecté » (figure 3). C'est un Internet multidimensionnel qui combine les champs de l'IdO et du Big data [MON 2015].

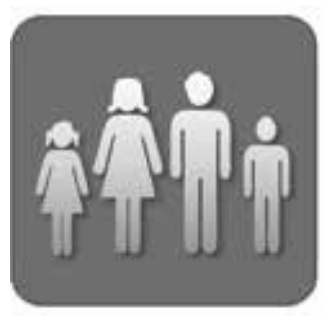

Personnes

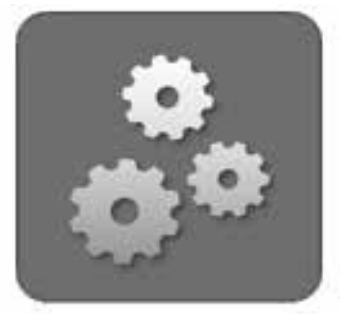

Processus

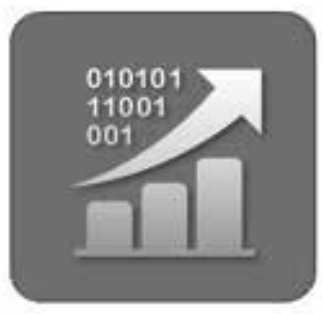

Données

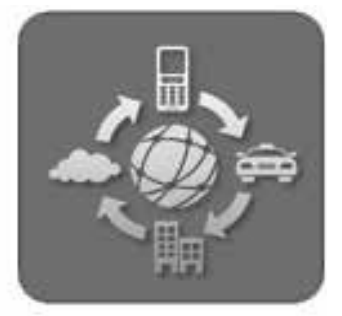

Objets

Figure 3. Internet of Everything

«Personnes : Connexion des personnes de manière plus pertinente et avec davantage de valeur.

Processus : Fournir la bonne information à la bonne personne (ou à la machine) au bon moment.

Données : S'appuyer sur les données pour faire ressortir les informations les plus utiles à la prise de décision. Objets : Dispositifs physiques et objets connectés à l'Internet pour une prise de décision intelligente. » [Source CIS 2013, tiré de MON 2015].

\section{Enjeux socio-économiques de l'IdO}

Les applications de l'IdO touchent pratiquement aujourd'hui toute la vie quotidienne :

- La santé et les systèmes de télésurveillance pour aider les personnes.

- L'agriculture connectée pour optimiser l'usage de l'eau.

- Les véhicules connectés aident à optimiser la gestion du trafic urbain.

- Les appareils électroménagers connectés aident à optimiser la consommation et la distribution de l'énergie électrique.

- Montre connectée pour le bien-être et le sport.

- Etc.

Ces exemples d'applications montrent que l'IdO est intégré dans notre vie quotidienne de plus, il améliore la qualité de vie des personnes [BOU 2017]. Il engendre un nouveau marché en créant de nouveaux emplois et métiers. Il aide également à la croissance pour les entreprises, et donne un élan pour

\footnotetext{
${ }^{2}$ http://www.cisco.com/c/dam/en_us/solutions/industries/docs/gov/everything-for-cities.pdf 
la compétitivité. Selon la GSMA [GSM], l'IdO est une industrie en pleine expansion à tous les niveaux matériels et logiciels qui devrait apporter aux opérateurs mobiles un revenu confortable autour de $\$ 1200$ milliards vers 2020 .

\section{Les enjeux et les défis de l'IdO}

L'IdO touche à tous les domaines de la co-construction des savoirs et des connaissances, de la gestion des entreprises et des administrations en passant par les technologies éducatives et hyperUrbaines, il transforme notre relation localisée en relation globalisée en dépassant les frontières géographiques [SAL 2012, 2014].

L'IdO est au centre de tous les développements technologiques modernes où la question des interfaces, des protocoles dans une situation stratégique majeure, est posée. Toute information, qu'elle soit textuelle, sonore ou visuelle, mais aussi gestuelle est en interaction permanente avec l'ensemble des autres, elle devient réticulaire. L'IdO transforme internet en HyperRéseau mondialisé dont tous les points formeraient autant de nœuds d'information possible sans frontière.

Les enjeux en terme de programmation informatique sont immenses. De nouvelles méthodes de conception, de développement, de débogage et de maintenance doivent voir le jour. À partir de langages symboliques innovants, les informaticiens de demain transformeront de simples lignes de code en agents autonomes capables d'exister dans la complexité des systèmes ubiquitaires et distribués, pour s'adapter aux demandes des utilisateurs, afin de recommander de nouveaux usages voir même de les accompagner dans l'évolution de leurs modes d'existence.

Ces bouleversements à la fois technologiques, sociétaux et humains soulèvent de nombreuses questions concernant la mutation sociétale et économique, mais également pour la recherche, la créativité et l'innovation. Il est important de penser à construire de nouveaux instruments pour un devenir technique au service du développement humain.

Un défi majeur pour l'IdO à la fois technique et utile, c'est de pouvoir gérer l'hétérogénéité technologique et des normes d'objets couplées à des multitudes de besoins d'applications et des usages en terme de services de sécurité. Sachant que ces besoins peuvent évoluer dans le temps selon le contexte et le goût. En effet, comment assurer l'authentification individuelle de plusieurs millions d'objets hétérogènes, dotés des technologies de communication hétérogènes, à travers des domaines administratifs multiples ? [VER 2011]. Ceci soulève des problèmes de gestion et de sécurité des objets dans un milieu hétérogène au niveau physique et logique. Il est donc important d'instaurer des politiques de sécurité claires, adaptatives selon le contexte d'utilisation et d'établir les responsabilités des faits et des actions sur l'environnement physique des objets. "En effet, l'Internet des objets est un système complexe dans lequel interagissent des personnes avec un écosystème technologique à base d'objets intelligents à travers des processus complexes. Les interactions de ces quatre composantes de l'IdO (personnes, objets intelligents, écosystème technologique, processus) font émerger une dimension systémique à la sécurité de l'IdO » [CHA 2012]. Par la suite, les tensions sur la sécurité de l'IdO sont créées lors de l'interaction des objets intelligents avec son environnement. Ces tensions (confiance, responsabilité, identification et auto-immunité) sont caractérisées par une dimension cognitive et systémique induite par l'autonomie grandissante des objets [CHA 2012].

L'IdO soulève une quantité de problèmes épistémologiques et sociotechniques : liberté-contrôle, autorité-indépendance, associativité-unicité, automatisme-maitrise, actions-interactions, contextualisation-décontextualisation, adaptativité-intégrité, etc. Nous devrons désormais apprendre à « penser à la fois le mobile, le flou, l'incertain, le proche et le lointain ${ }^{3} »$.

\footnotetext{
3 Jean-Pierre Balpe, « Des hypertextes à l'hypermonde » (http://nt2.uqam.ca/fr/actualites/des-hypertextes-l'hypermonde, consulté le 6/12/2016). 
L'enjeu du futur est de répondre de manière aussi créative que possible, aux inquiétudes anthropologiques, aux changements de régime climatiques, de la biodiversité, aux implications industrielles et à la transition énergétique, aux implications biopolitiques ainsi qu'éthiques d'accessibilité, de diversité culturelle, de redéfinitions de la notion de « données personnelles ». Mais c'est aussi poser la question de la dissémination des technologies intellectuelles comme contrepouvoirs dans une société datacentrique, en proposant des modèles d'analyses, des cadres conceptuels à la hauteur de ce qui s'avance, des méthodes de conception et des règles d'usages reconnus par l'ensemble des usagers, par les nouvelles intelligences collectives d'usages.

\section{Enjeux pour les entreprises et les chercheurs}

L'IdO croît sans cesse : " la mémoire invisible » du web basée sur les traces laissées par les utilisateurs, elles sont enregistrées dans des bases de données et analysées par le biais de méthodes statistiques et autres. Il nous semble qu'il est important que les acteurs publics ou privés saisissent l'opportunité de l'évolution de l'IdO et de cette mémoire pour proposer des approches originales pour bien être de l'humanité.

- Pour les entreprises, il s'agit de comprendre les comportements des usagers et les tendances des consommateurs pour proposer des services et des produits adaptés.

- Pour les acteurs politiques, ils peuvent suivre les tendances des sociétés pour ajuster leurs modes d'organisation, et leurs valeurs, adaptant leurs programmes et leur mode de fonctionnement.

- Pour les chercheurs en Sciences Humaines et Sociales (SHS), ils disposent de moyens d'observation fantastique « totaux » qui promettent "de révolutionner les méthodologies classiques en effaçant le clivage entre micro et macro, entre qualitatif et quantitative" [RIE 2010].

- Pour les chercheurs en Sciences (informatique, mathématiques, etc.), ils disposent d'une quantité de données pour tester la fiabilité, la rapidité et d'inventer des algorithmes.

- Pour les chercheurs en IHM, ils disposent de données et d'outils qui leur permettent d'imaginer et de créer des artefacts esthétiques et compréhensibles pour visualiser des données arrivées de manière quasicontinue [PIN 2017].

"La quantité et la richesse des données laisseraient penser que l'on peut passer, par une sorte de " zoom », de l'ensemble à l'individu et de la moyenne à l'idiosyncrasie » [RIE 2010]. Les traitements et l'analyse des traces numériques et des données produites par l'IdO sont en train de changer à la fois les modes de production et de communication de connaissances par les chercheurs, mais également l'organisation de la recherche, son économie et son rôle dans la société [RIE 2010].

\section{Objectifs de la revue}

Dans un monde « " hyperconnecté » via des objets connectés où les usagers sont à la fois émetteurs et récepteurs des données, l'IdO ouvre des champs nouveaux à explorer pour les sciences de l'information et de la communication pour étudier d'une part les enjeux sociétaux de ces nouveaux bouleversements technologiques et numériques et d'autre part analyser si les objets connectés répondent à des besoins d'usagers de plus en plus exigeants en matière de service, de communication et d'information.

Cette revue consacrée à l'Internet des objets traite d'une part de la réalité industrielle et technologique des objets connectés comme la gestion de l'entreprise, l'e-administration, l'e-gouvernement, mais aussi gestuelle : podomètre, direction du regard, GPS, etc., et d'autre part les impacts des objets connectés dans la vie quotidienne dans les domaines suivant : la santé, l'habitat, l'automobile, l'assurance, etc. 
La revue a pour objectif de valoriser les recherches et les réalisations basées sur les technologies numériques et les objets connectés, leurs usages quotidiens et leurs impacts socio-économiques. Les thématiques de la revue (non exhaustives) sont :

\section{IdO et Société}

- IdO et l'Ethiques

- IdO et le droit

- IdO et la confiance

- IdO et l'acceptation

- IdO et Interactions sociales

- IdO et E-gouvernement, E-économique, E-marketing

- IdO : Méthodologies de conception

- IdO : Modèles d'analyse

- Etc.

\section{IdO et Innovation technologique}

- Internet of EveryThing

- Web des objets

- Interface des objets

- Innovation et usages technologiques

- Environnements communicants et augmentés

- Écosystèmes d'informations et d'internet des objets

- Objets connectés, communicants

- Réseau d'objets numériques et physiques

- Normes et standards

- Big data

- IdO et data Science

\section{Nouveaux usages et nouveaux services :}

- IdO : Applications de santé

- IdO : Smart city

- IdO : Applications environnementales

- IdO : Surveillance de la circulation

- IdO : Croissance durable

- IdO : Smart Grid

- IdO : Gestion des données

- IdO : Sécurité, l'identité et la vie privée

- IdO : Aide après sinistre

- Etc 


\section{Bibliographie}

[BOU 2017] BOUHAÏ N., "Internte des objets : des objets envahissants ou indispensables", dans Bouhaï N. et Saleh I., (dir.) “Internet des objets : Évolutions et Innovations”, ISTE Éditions Londres, Mai 2017.

[BOY 2012] BOYD D., CTAWFORD K., Critical Question for Big Data, article consulté le 2/02/2017 site https://people.cs.kuleuven.be/ bettina.berendt/teaching/ViennaDH15/boyd_crawford_2012.pdf

[CHA 2012] CHALLAL Y., "Sécurité de l'Internet des Objets : vers une approche cognitive et systémique”, HDR, Juin 2012, UTC.

[CIS 2013] Cisco, "Value of the Internet of Everything for Cities", States ans Countries, 2013.

[CLU 2010] Cluster of European Research Projects on the Internet of Things, Vision and Challenges for Realising the Internet of Things, March 2010.

[COM 2009] Commission des Communautés Européennes, L'Internet des Objets : un plan d'action pour l'Europe, 2009.

[INT 2005] International Telecommunication Union, "Ubiquitous Network Societies: their impact on the telecommunication industry", ITU Workshop on Ubiquitous Network Societies, April 2005.

[GSM] GSMA http://www.gsm.org

[MAS 15] « Master In Data Science », disponible à l'adresse : https ://www.city.ac.uk/ courses/postgraduate/data-sciencemsc, consulté le 6 février 2017.

[MAV 2003] MAVROMMATI I., KAMEAS A., " The evolution of objects into hyper-objects: will it be mostly harmless?», Personal and Ubiquitous Computing. 2003. Disponible sur : http://dx.doi.org/10.1007/s00779- 003-0223-1

[MON 2015] Institut Montaigne, "Big data et objets connectés Faire de la France un champion de la révolution numérique », Rapport Avril 2015.

[PIN 2017] PINTO A. L., GONZALES-AGUILAR A., DUTRA M. L., SEMELER A. R. M., DENISCZWICZ, CLOSEL, C.,

"La visualisation de l'information pour l'Internet des objets", dans Bouhaï N. et Saleh I., (dir.) "Internet des objets : Évolutions et Innovations”, ISTE Éditions Londres, Mai, 2017.

[RIE 2010] REIDER, B., "Pratiques informationnelles et analyse des traces numériques : de la représentation à l'intervention", Etudes de communication, 2010, №35, p.91-104, site : https://edc.revues.org/2249, consulté le $03 / 02 / 2017$.

[RIE 2012] REIDER, B. \& RÖHLE, T. "Digital methods: five challenges", In D.M BERRY (Ed.) Understanding digital humanities. Houndmills: Palgrave Macmillan, p.67- 84.

[ROX 2017] ROXIN, I., BOUCHEREAU A., "Ecosystème de l'Internet des Objets", dans Bouhaï N. et Saleh I., (dir.) "Internet des objets : Évolutions et Innovations ”, ISTE Éditions Londres, Mai 2017.

[SAL 2012] SALEH I., HACHOUR H., « Le numérique comme catalyseur épistémologique », Revue Française des Sciences de l'Information et de la Communication, $\mathrm{N}^{\circ} 1,2012 \mathrm{http} / / / \mathrm{rfsic}$.revues.org/168,

[SAL 2014] SALEH I., HACHOUR H. et BOUHAI N., « Les frontières numériques » L'Harmmattan, septembre 2014, 260 pages.

[SEY et al. 2015] SEYDOUX N., BEN ALAYA M., HERNANDEZ N., MONTEIL T., HAEMMERLE O,. "Sémantique et

Internet des objets : d'un état de l'art à une ontologie modulaire", 26es Journées francophones d'Ingénierie des Connaissances, Jun 2015, Rennes, France. https://hal.archives-ouvertes.fr/IC-2015/hal-01166052, consulté le 3.03.2017.

[THE 2013] THEBAULT P., « La conception à l'ère de l'Internet des Objets : modèles et principes pour le design de produits aux fonctions augmentées par des applications", Thèse soutenue le 31 mai 2013, ParisTech.

[VER 2011] VERMESAN, O., et al. "Internet of Things Strategic Research Roadmap", Cluster of European Research Projects on the Internet of Things, 2011.

[WEI 91] WEISER M., « The computer for the XXIe century », Scientific American, vol. 265, n 3, p. 3- 11, 1991.

[WEI 93] WeISER M., « Hot Topics : Ubiquitous Computing » IEEE Computer, octobre 1993.

[WOO] WOOD L., "Today, the Internet, tomorrow-the Internet of Things", ComputerWorld,http://www.computerworld.com/s/article/9221614/Today_the_Internet_tomorrow_the_I nternet_of Things, November 2011. 